\title{
Perturbations preserving conditioned invariant subspaces *
}

\author{
Albert Compta, Josep Ferrer and Marta PeÑa \\ Departament de Matemàtica Aplicada I. \\ E.T.S. Enginyeria Industrial de Barcelona. UPC \\ Diagonal 647. 08028 Barcelona. Spain \\ Telephone: 34-934016549, Fax: 34-934011713 \\ albert.compta@upc.edu, josep.ferrer@upc.edu, marta.penya@upc.edu
}

\begin{abstract}
Given the set of matrix pairs $\mathcal{M} \subset$ $M_{m, n}(\mathbb{C}) \times M_{n}(\mathbb{C})$ keeping a subspace $S \subset \mathbb{C}^{n}$ invariant, we obtain a miniversal deformation of a pair belonging to an open dense subset of $\mathcal{M}$. It generalizes the known results when $S$ is a supplementary subspace of the unobservable one.
\end{abstract}

Keywords-Conditioned invariant subspaces, Miniversal deformation, Stratified manifold, Vertical pairs of matrices.

\section{INTRODUCTION}

This paper contributes to the study of versal deformations when square matrices or pairs of matrices are considered, together with invariant subspaces. More specifically, we consider perturbations of a pair keeping a certain subspace invariant. Versal deformations were introduced by Arnold in [1] to study the variations of the invariants of a square matrix when its entries are perturbed. Thanks to a natural generalization contained in [16], the same technique has been applied to other cases, such as perturbations of pairs of matrices representing linear systems ([9], [10]).

Invariant subspaces play a key role both in matrix theory (see [14]) and linear control systems (see [17]), where they are often called "conditioned" invariant subspaces. For instance, in [17] invariant subspaces are used in control problems such as Disturbance Decoupling or Output Regulation. From a theoretical point of view, the differentiable structure of the set of invariant subspaces of a square

\footnotetext{
${ }^{*}$ Manuscript received July 12, 2010. This work was partially supported by DGICYT DGICYT MTM2007-67812C02-02.
}

matrix has been studied in [15] and that of conditioned invariant subspaces of a pair in [12] and [13].

In the context of versal deformations, it seems natural to consider the situation when both a matrix and an invariant subspace are involved and both or one of the elements of this couple is perturbed. So, in [7] one studies the perturbation of a square matrix preserving an invariant subspace. In particular, this perturbation gives all the solutions of the Carlson problem, and hence explicit realizations can be obtained (see [5]).

Here we generalize [7] to linear systems. That is to say, we consider the perturbation of a pair of matrices preserving a given conditioned invariant subspace: we obtain the equations of a miniversal deformation (i.e., minimal dimensional versal deformation).

More specifically, we let $M_{p, q}(\mathbb{C})$ denote the set of complex matrices having $p$ rows and $q$ columns. If $p=q$, we simply write $M_{p}(\mathbb{C})$, and $G l(p)$ denotes the group of nonsingular $p \times p$ matrices. If $p \leq q$, we identify $\mathbb{C}^{p}$ as the subspace $\mathbb{C}^{p} \times\{0\} \subset \mathbb{C}^{q}$, that is, the vectors having $q-p$ zero trailing entries. We consider the set $\mathcal{M} \subset M_{n+m, n}(\mathbb{C})$ of vertically embedded pairs of matrices having $\mathbb{C}^{d}$ as a conditioned invariant subspace (Definition 0.1). Two pairs of matrices will be called equivalent if they are block-similar and the change of basis in the state space $\mathbb{C}^{n}$ induces an automorphism in $\mathbb{C}^{d}$ (Definition 0.2). Our aim is to study these equivalence classes and their variations when a pair is perturbed preserving $\mathbb{C}^{d}$ as conditioned invariant subspace.

Section 3 is devoted to study the differentiable 
structure of $\mathcal{M}$, which is the starting point to apply Arnold's techniques (see [1]). If we fix a basis adapted to $\mathbb{C}^{d} \subset \mathbb{C}^{n} \subset \mathbb{C}^{n+m}$, in Proposition 0.5 we prove that the elements of $\mathcal{M}$ are those of the form

$$
\mathcal{A}=\left(\begin{array}{cc}
A_{1} & A_{3} \\
F C_{1} & A_{2} \\
\hline C_{1} & C_{2}
\end{array}\right) .
$$

Notice that for the particular case of square matrices in [7], one obtains simply $\left(\begin{array}{cc}A_{1} & A_{3} \\ 0 & A_{2}\end{array}\right)$. Hence the generalization to pairs of matrices is non-trivial. In fact, we prove that $\mathcal{M}$ can be differentially stratified by $\operatorname{rank} C_{1}$ (Theorem 0.7) and the above equivalence classes are the orbits under the action on each stratum of a suitable group (Definition 0.8).

In Section 4 we obtain the equation of a miniversal deformation of a pair belonging to the maximal stratum $\mathcal{M}^{*}$, which is an open dense set in $\mathcal{M}$. Then the equations of a miniversal deformation are obtained in Theorem 0.14.

As an example, we see that they generalize the ones obtained in [9] when $\mathbb{C}^{d}$ is a supplementary subspace of the unobservable of $\mathcal{A}$.

\section{Preliminaries}

We will deal with matrices of the form $\left(\begin{array}{c}A \\ C\end{array}\right) \in$ $M_{n+m, n}(\mathbb{C})$, which we will identify with the pairs of matrices $(C, A)$, and which will be simply denoted as $\mathcal{A}$ if no confusion is possible.

We are interested in pairs $(C, A)$ having a fixed subspace $S \in \mathbb{C}^{n}$ as "invariant", in the sense of [2].

Definition 0.1 A subspace $S \subset \mathbb{C}^{n}$ is $(C, A)$ invariant or conditioned invariant if $A(S \cap \operatorname{Ker} C) \subset$ $S$.

When conditioned invariant subspaces are involved, the usual block similarity between pairs of matrices is restricted in a natural way:

Definition 0.2 Given two pairs of matrices $(C, A),\left(C^{\prime}, A^{\prime}\right) \in M_{n+m, n}(\mathbb{C})$ having $S \subset \mathbb{C}^{n}$ as a conditioned invariant subspace, we say that they are $S$-equivalent (or simply equivalent if no confusion is possible) if (i) the pairs are block-similar, that is, $A^{\prime}=$ $P A P^{-1}+R C P^{-1}, C^{\prime}=Q C P^{-1}$, where $P \in$ $G l(n), Q \in G l(m), R \in M_{n, m}(\mathbb{C})$,

(ii) and the change of basis $P$ preserves $S$.

\section{PAirs OF MATRICES HAVING A $(C, A)$-InVariant fiXed Subspace}

In this section, we characterize the elements of the set $\mathcal{M}$ formed by the pairs of matrices having the subspace $S \subset \mathbb{C}^{n}$ as a conditioned invariant. Moreover, we show that these elements form a stratified manifold and finally we describe a Lie group that acts on $\mathcal{M}$ in such a way that the orbits are just the equivalence classes in Definition 0.2.

The subspace $S$ may be identified with $\mathbb{C}^{d}$ by considering "adapted basis":

Definition 0.3 Let us consider a subspace $S \subset$ $\mathbb{C}^{n}, \operatorname{dim} S=d$. A basis of $\mathbb{C}^{n}$ whose $d$ first vectors form a basis of $S$ is called an adapted basis to the subspace.

In the above conditions, we will assume that the matrices $A$ and $C$ are block-partitioned into

$$
A=\left(\begin{array}{cc}
A_{1} & A_{3} \\
A_{4} & A_{2}
\end{array}\right), \quad C=\left(\begin{array}{ll}
C_{1} & C_{2}
\end{array}\right),
$$

where $A_{1} \in M_{d}(\mathbb{C}), \quad C_{1} \in M_{m, d}(\mathbb{C})$.

Now we define and characterize the set $\mathcal{M}$ :

Definition 0.4 Given $S=\mathbb{C}^{d} \subset \mathbb{C}^{n}$, let $\mathcal{M}=\left\{(C, A) \in M_{n+m, n}(\mathbb{C}): A(S \cap \operatorname{Ker} C) \subset S\right\}$ (that is to say, $S$ is $(C, A)$-invariant.)

Proposition 0.5 Let $(C, A) \in M_{m, n}(\mathbb{C}) \times M_{n}(\mathbb{C})$.

(1) $(C, A) \in \mathcal{M}$ if and only if there is a basis adapted to $S$ such that the pair becomes

$$
\left(\begin{array}{c}
\bar{A} \\
\bar{C}
\end{array}\right)=\left(\begin{array}{cc}
\bar{A}_{1} & \bar{A}_{3} \\
0 & \bar{A}_{2} \\
\hline C_{1} & C_{2}
\end{array}\right),
$$

with $\bar{A}_{1} \in M_{d}(\mathbb{C}), \bar{C}_{1} \in M_{m, d}(\mathbb{C}), d=\operatorname{dim} S$. 
(2) Equivalently, if the pair $(C, A)$ has the form (in any adapted basis)

$$
\left(\begin{array}{c}
A \\
C
\end{array}\right)=\left(\begin{array}{cc}
A_{1} & A_{3} \\
F C_{1} & A_{2} \\
\hline C_{1} & C_{2}
\end{array}\right),
$$

for some $F \in M_{n-d, m}(\mathbb{C})$.

Proof.

(1) See, for example, [4].

(2) The elements of $\mathbb{C}^{d} \cap \operatorname{Ker} C$ have the form $\left(\begin{array}{l}x \\ 0\end{array}\right)$ with $C_{1} x=0$. Thus, $A\left(\mathbb{C}^{d} \cap \operatorname{Ker} C\right) \subset$ $\mathbb{C}^{d}$. is verified if and only if $C_{1} x=0$ implies $A_{4} x=0$, that is, $\operatorname{Ker} C_{1} \subset \operatorname{Ker} A_{4}$. But this last condition is equivalent to $\operatorname{Im} A_{4}^{t} \subset \operatorname{Im} C_{1}^{t}$; or, equivalently, $A_{4}^{t}=C_{1}^{t} F^{t}$ for some $F \in$ $M_{n-d, m}(\mathbb{C})$.

In order to study the differentiable structure of $\mathcal{M}$, let us consider the set

$$
\mathcal{N}=\left\{(D G, D): D \in M_{d, m}(\mathbb{C}), G \in M_{m, l}(\mathbb{C})\right\} .
$$

In general, $\mathcal{N}$ is not a manifold. For example, $\{(x y, x): x \in \mathbb{R}, y \in \mathbb{R}\}=\{(0,0)\} \cup\{(z, x): x \neq$ $0, z \in \mathbb{R}\}$. However, we can stratify $\mathcal{N}$ by means of $\operatorname{rank} D$ :

\section{Lemma 0.6 The set}

$$
\begin{gathered}
\mathcal{N}_{r}=\left\{(D G, D): D \in M_{d, m}(\mathbb{C}), G \in M_{m, l}(\mathbb{C}),\right. \\
\operatorname{rank} D=r\}
\end{gathered}
$$

is a manifold of dimension $r(l+m+d-r)$.

\section{Proof.}

Let $M_{d, m}^{r}(\mathbb{C})$ be the set of matrices of $M_{d, m}(\mathbb{C})$ with rank $r$, which is a manifold of dimension $d m-(d-r)(m-r)$ (see, for example [3]). If $D_{0} \in M_{d, m}^{r}(\mathbb{C})$, let $\mathcal{U}_{D_{0}}$ be an open neighborhood in $M_{d, m}^{r}(\mathbb{C})$. Without loss of generality we can assume that $\operatorname{Im} D_{0}$ is spanned by its first $r$ columns and that this property also holds for all $D \in \mathcal{U}_{D_{0}}$.

Then, for any $D \in \mathcal{U}_{D_{0}}$ and each column $g_{j}$ of $G$, $1 \leq j \leq l, D g_{j}$ is parameterized by the top $r$ coefficients of $g_{j}$. Therefore, a coordinate chart of the matrices $(D G, D)=\left(D g_{1}, \ldots, D g_{l}, D\right)$ is formed by $\mathcal{U}_{D_{0}}$ and the first $r$ rows of $G$, and the dimension of $\mathcal{N}_{r}$ is $d m-(d-r)(m-r)+r l$.

Therefore, $\mathcal{M}$ is a stratified differentiable manifold:

Theorem 0.7 Let $\mathcal{M}_{r} \subset \mathcal{M}$ defined by:

$\mathcal{M}_{r}=\left\{\left(\begin{array}{ll}A_{1} & A_{3} \\ A_{4} & A_{2} \\ \hline C_{1} & C_{2}\end{array}\right) \in \mathcal{M}: \operatorname{rank} C_{1}=r\right\}$,

$\mathcal{M}=\bigcup_{0 \leq r \leq r^{*}} \mathcal{M}_{r}$, where $r^{*}=\min (d, m)$.

(1) $\mathcal{M}_{r}$ is a manifold of dimension

$$
\sigma_{r}=d^{2}+(n-d)(n+m)+r(n+m-r) .
$$

(2) $\mathcal{M}=\bigcup_{r} \mathcal{M}_{r}$ is a stratified differentiable manifold.

(3) The stratum

$$
\mathcal{M}^{*}=\left\{\mathcal{A} \in \mathcal{M}: \operatorname{rank} C_{1}=r^{*}\right\},
$$

where $r^{*}=\min (d, m)$, is an open dense subset in $\mathcal{M}$.

As a first consequence, the equivalence classes in Definition 0.2 are the orbits in $\mathcal{M}$ under the action of a suitable Lie group. In a natural way, we consider change of bases in $M_{n+m, n}(\mathbb{C})$ which preserve the chain of subspaces $\mathbb{C}^{d} \subset \mathbb{C}^{n} \subset \mathbb{C}^{n+m}$.

Definition 0.8 Let $\mathcal{G} \subset G l(n+m)$ be defined by

$$
\begin{gathered}
\mathcal{G}=\left\{\mathcal{P}=\left(\begin{array}{cc|c}
P_{1} & P_{3} & R_{1} \\
0 & P_{2} & R_{2} \\
\hline 0 & 0 & Q
\end{array}\right) \in G l(n+m),\right. \\
\left.P_{1} \in G l(d), P_{2} \in G l(n-d), Q \in G l(m)\right\} .
\end{gathered}
$$

It is a straightforward computation that $\mathcal{G}$ is a subgroup of $G l(n+m)$ and that the following result holds.

Proposition 0.9 The natural action of the subgroup $\mathcal{G} \subset G l(N+n)$ on the differentiable manifold $M_{n+m, n}(\mathbb{C})$ can be restricted to $\mathcal{M}_{r}$. That is, if $\mathcal{P} \in \mathcal{G}$ and $\mathcal{A} \in \mathcal{M}_{r}$, then

$$
\mathcal{P} * \mathcal{A}=
$$

$\left(\begin{array}{cc|c}P_{1} & P_{3} & R_{1} \\ 0 & P_{2} & R_{2} \\ \hline 0 & 0 & Q\end{array}\right)\left(\begin{array}{cc}A_{1} & A_{3} \\ F C_{1} & A_{2} \\ \hline C_{1} & C_{2}\end{array}\right)\left(\begin{array}{cc}P_{1} & P_{3} \\ 0 & P_{2}\end{array}\right)^{-1} \in$ 
$\mathcal{M}_{r}$.

If we denote by $\mathcal{O}_{\mathcal{A}}$ the orbit of $\mathcal{A} \in \mathcal{M}_{r}$ under the action of $\mathcal{G}$, then $\mathcal{O}_{\mathcal{A}}$ is the equivalence class of $\mathcal{A}$ with respect to the equivalence relation in Definition 0.2. Hence, it is a locally closed submanifold of $\mathcal{M}_{r}$, with the boundary consisting of the union of orbits of strictly smaller dimension.

\section{Miniversal DEFormation PRESERVING A (C,A)-INVARIANT SUBSPACE}

In order to study the perturbations of a pair $(C, A)$ preserving a conditioned invariant subspace, we will use Arnold's techniques of the so-called versal deformations (that is, canonical forms of local differentiable families of perturbations). The starting point is the fact that the equivalence classes are orbits under the action of a Lie group, and hence they are submanifolds. Then versal/miniversal deformations can be obtained as submanifolds which are transverse/minitransverse to the orbit. We recall some general definitions and results which we will apply to $\mathcal{N}=\mathcal{M}^{*}$.

Definition 0.10 Let $\mathcal{N}$ be a manifold. An $l$ dimensional deformation of $\mathcal{A} \in \mathcal{N}$ is a differentiable map

$$
\varphi: \Lambda \longrightarrow \mathcal{N}
$$

where $\Lambda$ is a neighborhood of the origin in $\mathbb{C}^{l}$ and $\varphi(0)=\mathcal{A}$. The image $\varphi(\Lambda)$ is said to be a family of deformations of $\mathcal{A} \in \mathcal{N}$.

If there is a Lie group $\mathcal{G}$ acting on the differentiable manifold $\mathcal{N}, \mathcal{G} \times \mathcal{N} \longrightarrow \mathcal{N},(\mathcal{P}, \mathcal{A}) \longmapsto \mathcal{P} * \mathcal{A}$, a deformation is called "versal" if any other deformation is induced from it in the following sense:

Definition 0.11 Let $\mathcal{N}$ be a manifold and $\mathcal{G}$ a Lie group acting on it. A deformation of $\mathcal{A} \in \mathcal{N}$, $\varphi: \Lambda \longrightarrow \mathcal{N}$ is called versal if, given any other deformation of $\mathcal{A} \in \mathcal{N}, \psi: \Gamma \longrightarrow \mathcal{N}$, there is a neighborhood of the origin $\Gamma^{\prime} \subset \Gamma$, a differentiable map $\rho: \Gamma^{\prime} \longrightarrow \Lambda$ and a deformation of the identity $I \in \mathcal{G}, \delta: \Gamma^{\prime} \longrightarrow \mathcal{G}$ such that

$$
\psi(\tau)=\delta(\tau) * \varphi(\rho(\tau)), \forall \tau \in \Gamma^{\prime} .
$$

It is called miniversal if it has the minimal dimension among the versal deformations.
Remark 0.12 It is enough to compute a miniversal deformation of a point of the orbit; then, a miniversal deformation of any other point of the same orbit is induced from it by means of the group action.

We now recall the key relation between "versality" and "transversality", proved in [1] for square matrices, and which can be generalized (for example [16]) to the cases like the above one, where the equivalence classes are submanifolds given as orbits under the action of a Lie group.

Proposition 0.13 Let $\mathcal{N}$ be a manifold, with a Lie group $\mathcal{G}$ acting on it. Let $\mathcal{A} \in \mathcal{N}, \mathcal{O}_{\mathcal{A}}$ be its $\mathcal{G}$ orbit and $\mathcal{L} \subset \mathcal{N}$ be a submanifold minitransverse to $\mathcal{O}_{\mathcal{A}}$ at $\mathcal{A}$, that is to say: $T_{\mathcal{A}} \mathcal{N}=T_{\mathcal{A}} \mathcal{L} \oplus T_{\mathcal{A}} \mathcal{O}_{\mathcal{A}}$, where $T_{\mathcal{A}}(\cdot)$ means the tangent space at $\mathcal{A}$. Then any local parametrization of $\mathcal{L}$ at $\mathcal{A}$ is a miniversal deformation of $\mathcal{A}$ in $\mathcal{N}$.

Thus, we have our main result for a generic pair $\mathcal{A} \in \mathcal{M}^{*} \subset \mathcal{M}$ :

Theorem 0.14 Let $\mathcal{A}=(C, A) \in M_{n+m, n}(\mathbb{C})$ be a pair of matrices and $S \subset \mathbb{C}^{n}$ a d-dimensional $(C, A)$-invariant subspace:

$$
\mathcal{A}=\left(\begin{array}{c}
A \\
C
\end{array}\right)=\left(\begin{array}{cc}
A_{1} & A_{3} \\
0 & A_{2} \\
\hline C_{1} & C_{2}
\end{array}\right)
$$

(see Proposition 0.5). Assume $\mathcal{A} \in \mathcal{M}^{*}$, that is to say, $\operatorname{rank} C_{1}=r^{*}=\min (d, m)$. Then the following statements hold.

(i) If $m \leq d$, a miniversal deformation of $\mathcal{A}$ preserving $S$ as conditioned invariant subspace is given by the linear submanifold $\mathcal{L} \subset \mathcal{M}$ formed by the matrices:

$$
\mathcal{A}+\left(\begin{array}{cc}
X_{1} & X_{3} \\
Z\left(C_{1}+Y_{1}\right) & X_{2} \\
\hline Y_{1} & Y_{2}
\end{array}\right)
$$

satisfying the conditions

(1) $X_{2} C_{2}^{*}+Z=0$,

(2) $Y_{1} C_{1}^{*}+Y_{2} C_{2}^{*}=0$,

(3) $X_{1} C_{1}^{*}+X_{3} C_{2}^{*}=0$,

(4) $X_{3} A_{2}^{*}-A_{1}^{*} X_{3}-C_{1}^{*} Y_{2}=0$,

(5) $X_{1} A_{1}^{*}+X_{3} A_{3}^{*}-A_{1}^{*} X_{1}-C_{1}^{*} Y_{1}=0$, 
(6) $X_{2} A_{2}^{*}-A_{3}^{*} X_{3}-A_{2}^{*} X_{2}-C_{2}^{*} Y_{2}=0$.

(ii) If $d<m$, without loss of generality we can assume $C_{1}^{t}=\left(I_{d}, 0\right)$. Then, a miniversal deformation of $\mathcal{A}$ in $\mathcal{M}$ is given by the set of matrices

$$
\mathcal{A}+\left(\begin{array}{cc}
X_{1} & X_{3} \\
Z\left(I_{d}+Y_{11}\right) & X_{2} \\
\hline Y_{1} & Y_{2}
\end{array}\right),
$$

where $Y_{1}=\left(\begin{array}{c}Y_{11} \\ Y_{12}\end{array}\right), \quad Y_{11} \in M_{d}(\mathbb{C})$, satisfying (2)-(6) above and

$\left(1^{\prime}\right) \quad X_{2} C_{2}^{*}+(Z, 0)=0$, with $Z \in$ $M_{n-d, r^{*}}(\mathbb{C})$,

Proof. Firstly, let us assume $m \leq d$. Then the subset $U$ of matrices $\bar{C}_{1} \in M_{m, d}(\overline{\mathbb{C}})$ having maximal rank is open and dense, and $F \bar{C}_{1}=0$ only if $F=0$. Hence, the map

$$
\begin{gathered}
\gamma: W \longrightarrow \mathcal{M}^{*}, \\
W=M_{d}(\mathbb{C}) \times M_{n-d}(\mathbb{C}) \times M_{d, n-d}(\mathbb{C}) \times U \times \\
M_{m, n-d}(\mathbb{C}) \times M_{n-d, m}(\mathbb{C}), \\
\gamma\left(\bar{A}_{1}, \bar{A}_{2}, \bar{A}_{3}, \bar{C}_{1}, \bar{C}_{2}, F\right)=\left(\begin{array}{cc}
\bar{A}_{1} & \bar{A}_{3} \\
F \bar{C}_{1} & \bar{A}_{2} \\
\hline C_{1} & C_{2}
\end{array}\right)
\end{gathered}
$$

is a parametrization of $\mathcal{M}^{*}$. Bearing in mind Proposition 0.13 , if $\mathcal{V} \subset \mathcal{M}^{*}$ is a sufficiently small neighborhood of $\mathcal{A}$, it is sufficient to prove that $\gamma^{-1}(\mathcal{V} \cap \mathcal{L})$ is minitransverse to $\gamma^{-1}\left(\mathcal{V} \cap \mathcal{O}_{\mathcal{A}}\right)$ at $\tilde{\mathcal{A}} \doteq \gamma^{-1}(\mathcal{A})$. In fact, we will prove that the first one is just the normal variety to the second one at $\tilde{\mathcal{A}}$ with respect to the natural scalar product: $\left\langle\left(X_{1}, X_{2}, \ldots\right),\left(X_{1}^{\prime}, X_{2}^{\prime}, \ldots\right)\right\rangle=\operatorname{trace} X_{1}^{*} X_{1}^{\prime}+$ trace $X_{2}^{*} X_{2}^{\prime}+\ldots$, where $X_{i}^{*}$ means the conjugatetranspose matrix of $X_{i}$.

It is a straightforward computation that the matrices in $\gamma^{-1}\left(\mathcal{V} \cap \mathcal{O}_{\mathcal{A}}\right)$ can be seen as the orbit of $\tilde{\mathcal{A}}$ under the action of $\mathcal{G}$ induced in a natural way from Proposition 0.9 as $\mathcal{P} * \tilde{\mathcal{A}}=\gamma^{-1}(\mathcal{P} * \mathcal{A})$. Then,

$$
\begin{gathered}
\mathcal{P} * \tilde{\mathcal{A}}=\left(P_{1} A_{1} P_{1}^{-1}+R_{1} C_{1} P_{1}^{-1},\right. \\
-R_{2} C_{1} P_{1}^{-1} P_{3} P_{2}^{-1}+\left(P_{2} A_{2}+R_{2} C_{2}\right) P_{2}^{-1}, \\
-\left(P_{1} A_{1}+R_{1} C_{1}\right) P_{1}^{-1} P_{3} P_{2}^{-1}+ \\
\left(P_{1} A_{3}+P_{3} A_{2}+R_{1} C_{2}\right) P_{2}^{-1}, Q C_{1} P_{1}^{-1}, \\
\left.-Q C_{1} P_{1}^{-1} P_{3} P_{2}^{-1}+Q C_{2} P_{2}^{-1}, R_{2} Q^{-1}\right) .
\end{gathered}
$$

Clearly, its tangent space at $\tilde{\mathcal{A}}$ is $\operatorname{Im} d \alpha_{I}$, where $d \alpha_{I}$ is the derivative at the identity $I \in \mathcal{G}$ of the map nference on Circuits, Systems, Signals

$\alpha: \mathcal{G} \longrightarrow W, \quad \alpha(\mathcal{P})=\mathcal{P} * \tilde{\mathcal{A}}$. Differentiating this map we have:

$$
\begin{gathered}
d \alpha_{I}(\dot{\mathcal{P}})=\left(\dot{P}_{1} A_{1}-A_{1} \dot{P}_{1}+\dot{R}_{1} C_{1},\right. \\
\dot{P}_{2} A_{2}+\dot{R}_{2} C_{2}-A_{2} \dot{P}_{2}, \\
-A_{1} \dot{P}_{3}+\dot{P}_{1} A_{3}+\dot{P}_{3} A_{2}+\dot{R}_{1} C_{2}-A_{3} \dot{P}_{2}, \\
\left.\dot{Q} C_{1}-C_{1} \dot{P}_{1},-C_{1} \dot{P}_{3}+\dot{Q} C_{2}-C_{2} \dot{P}_{2}, \dot{R}_{2}\right)
\end{gathered}
$$

for any $\dot{\mathcal{P}}$ belonging to $T_{I} \mathcal{G}$, that is, for $\dot{P}_{1} \in$ $M_{d}(\mathbb{C}), \dot{P}_{2} \in M_{n-d}(\mathbb{C}), \dot{P}_{3} \in M_{d, n-d}(\mathbb{C}), \dot{R}_{1} \in$ $M_{d, m}(\mathbb{C}), \dot{R}_{2} \in M_{n-d, m}(\mathbb{C}), \dot{Q} \in M_{m}(\mathbb{C})$.

Then, $\left(X_{1}, X_{2}, X_{3}, Y_{1}, Y_{2}, Z\right) \in\left(T_{\tilde{\mathcal{A}}} \gamma^{-1}\left(\mathcal{V} \cap \mathcal{O}_{\mathcal{A}}\right)\right)^{\perp}$ if and only if, for any $\dot{P}_{1}, \dot{P}_{2}, \dot{P}_{3}, \dot{R}_{1}, \dot{R}_{2}, \dot{Q}$ as above,

$$
\begin{gathered}
\operatorname{trace}\left(X_{1}^{*}\left(\dot{P}_{1} A_{1}-A_{1} \dot{P}_{1}+\dot{R}_{1} C_{1}\right)\right)+ \\
\operatorname{trace}\left(X_{2}^{*}\left(\dot{P}_{2} A_{2}+\dot{R}_{2} C_{2}-A_{2} \dot{P}_{2}\right)\right)+ \\
\operatorname{trace}\left(X_{3}^{*}\left(-A_{1} \dot{P}_{3}+\dot{P}_{1} A_{3}+\dot{P}_{3} A_{2}+\dot{R}_{1} C_{2}-A_{3} \dot{P}_{2}\right)\right)+ \\
\operatorname{trace}\left(Y_{1}^{*}\left(\dot{Q} C_{1}-C_{1} \dot{P}_{1}\right)\right)+ \\
\operatorname{trace}\left(Y_{2}^{*}\left(-C_{1} \dot{P}_{3}+\dot{Q} C_{2}-C_{2} \dot{P}_{2}\right)\right)+\operatorname{trace}\left(Z^{*} \dot{R}_{2}\right)=0 \\
\text { or, equivalently, } \\
\operatorname{trace}\left(\left(X_{1} A_{1}^{*}+X_{3} A_{3}^{*}-A_{1}^{*} X_{1}-C_{1}^{*} Y_{1}\right) \dot{P}_{1}^{*}\right)+ \\
\operatorname{trace}\left(\left(X_{3} A_{2}^{*}-A_{1}^{*} X_{3}-C_{1}^{*} Y_{2}\right) \dot{P}_{3}^{*}\right)+ \\
\operatorname{trace}\left(\left(X_{1} C_{1}^{*}+X_{3} C_{2}^{*}\right) \dot{R}_{1}^{*}\right)+ \\
\operatorname{trace}\left(\left(X_{2} A_{2}^{*}-A_{3}^{*} X_{3}-A_{2}^{*} X_{2}-C_{2}^{*} Y_{2}\right) \dot{P}_{2}^{*}\right)+
\end{gathered}
$$$$
\operatorname{trace}\left(\left(X_{2} C_{2}^{*}+Z\right) R_{2}^{*}\right)+\operatorname{trace}\left(\left(Y_{1} C_{1}^{*}+Y_{2} C_{2}^{*}\right) \dot{Q}^{*}\right)=0 \text {. }
$$

Hence, the matrices in $\left(T_{\tilde{\mathcal{A}}} \gamma^{-1}\left(\mathcal{V} \cap \mathcal{O}_{\mathcal{A}}\right)\right)^{\perp}$ are just characterized by $(1)-(6)$.

If $d<m$ and $C_{1}^{t}=\left(I_{d}, 0\right)$ then for any $\bar{C}_{1}=\left(\begin{array}{l}\bar{C}_{11} \\ \bar{C}_{12}\end{array}\right)$ in the vicinity of $C_{1}$ it holds that $\bar{C}_{11}$ is non-singular. So that: $\bar{F} \bar{C}_{1}=$ $\left(\begin{array}{ll}\bar{F}_{1} & \bar{F}_{2}\end{array}\right)\left(\begin{array}{c}\bar{C}_{11} \\ \bar{C}_{12}\end{array}\right)=\left(\bar{F}_{1}+\bar{F}_{2} \bar{C}_{12} \bar{C}_{11}^{-1}\right) \bar{C}_{11} \doteq$ $F_{1} \bar{C}_{11}$. Hence, the map

$$
\begin{gathered}
\gamma: M_{d}(\mathbb{C}) \times M_{n-d}(\mathbb{C}) \times M_{d, n-d}(\mathbb{C}) \times U \times \\
M_{m, n-d}(\mathbb{C}) \times M_{n-d, d}(\mathbb{C}) \longrightarrow \mathcal{M}^{*} \\
\gamma\left(\bar{A}_{1}, \bar{A}_{2}, \bar{A}_{3}, \bar{C}_{1}, \bar{C}_{2}, F_{1}\right)=\left(\begin{array}{cc}
\bar{A}_{1} & \bar{A}_{3} \\
F_{1} \bar{C}_{11} & \bar{A}_{2} \\
\hline C_{1} & C_{2}
\end{array}\right)
\end{gathered}
$$

is a local parametrization. Then, the proof works as above. 
Example 0.15 Let us consider the particular case when $S$ is a supplementary subspace of the unobservable one. Clearly, the pair can be taken in the usual Brunovsky-Kronecker form

$$
\mathcal{A}_{B K}=\left(\begin{array}{cc}
N & 0 \\
0 & J \\
\hline E & 0
\end{array}\right) .
$$

where $N$ is block-diagonal of nilpotent blocks, $J$ is a Jordan matrix, and $E$ a full rank block diagonal, with blocks having the form $(0, \ldots, 0,1)$. Hence, the equations in Theorem 0.14 become:

(1) $Z=0$,

(2) $Y_{1} E^{*}=0$,

(3) $X_{1} E^{*}=0$,

(4) $X_{3} J-N^{*} X_{3}-E^{*} Y_{2}=0$,

(5) $X_{1} N^{*}-N^{*} X_{1}-E^{*} Y_{1}=0$,

(6) $X_{2} J^{*}-J^{*} X_{2}=0$.

Let us remark that these equations are just the (I)-(V) in [9] (page 185). We point out that horizontal pairs are considered in [9]. Hence the matrices N, J, E in [9] correspond in our natation to $N^{*}, J^{*}, E^{*}$. In a similar way, the solutions $U_{1}^{1}, U_{2}^{1}, U_{1}^{2}, U_{2}^{2}, V_{1}^{1}$ and $V_{2}^{1}$ must be identified with $X_{1}, X_{3}, Z\left(C_{1}+Y_{1}\right), X_{2}, Y_{1}$ and $Y_{2}$ respectively, whereas $V_{1}^{2}$ and $V_{2}^{2}$ do not appear here due to our hypothesis of the pair of matrices having full rank.

Remark 0.16 The above example shows the quite surprising fact that the same perturbed pairs appear with the restriction or not that a fixed subspace $S$ is preserved as conditioned invariant. Indeed, from the results in [9] it follows that, when $S$ is a supplementary subspace of the unobservable one of the initial pair, then it is also conditioned invariant for all the perturbed pairs. In this sense our approach is a generalization of [9] for $S$ being any conditioned invariant subspace.

\section{References}

[1] V.I. Arnold, On Matrices Depending on Parameters, Uspekhi Mat. Nauk., 26 (1971), p. 101-114.
[2] G. Basile; G. Marro, Controlled and conditioned invariant subspaces in linear system theory, J. Optim. Theory Appl., 3 (1969), p. 306315.

[3] Th. BRocker; L. LANDER, Differentiable Germs and Catastrophes, Cambridge University Press, Cambridge (1975).

[4] A. Compta; J. Ferrer, On $(A, B)^{t}$ invariant Subspaces Having Extendible Brunovsky Bases, Linear Algebra Appl., 225 (1997), p. 185-201.

[5] A. Compta; J. Ferrer, Matricial Realizations of the Solutions of the Carlson Problem,Linear Algebra Appl., 353 (2002), p. 197206.

[6] A. Compta; J. Ferrer; M. PeÑA, Dimension of the Orbit of Marked Subspaces,Linear Algebra Appl., 379 (2004), p. 239-248.

[7] A. Compta; J. Ferrer; F. Puerta, Miniversal deformations of marked matrices, Linear Algebra Appl., 361 (2003), p. 181-201.

[8] A. Compta; U. Helmke; M. Peña; X. Puerta, Simultaneous Versal Deformations of Endomorphisms and Invariant Subspaces, Linear Algebra Appl., 413 (2006), p. 303-318.

[9] J. Ferrer; M.I. García; F. Puerta, Brunovsky Local Form of a Holomorphic Family of Pais of Matrices, Linear Algebra Appl., 253 (1997), p. 175-198.

[10] J. Ferrer; M.I. García; F. Puerta, Regularity of the Brunovsky-Kronecker Stratification, SIAM Journal on Matrix Analysis Appl. Vol. 21, n. 3(2000), p. 724-742.

[11] J. Ferrer; F. Puerta, Versal Deformations of Invariant Subspaces, Linear Algebra Appl., 332-334 (2001), p. 569-582.

[12] J. Ferrer; F. Puerta; X. Puerta, Differentiable Structure of the Set of Controllable $(A, B)^{t}$-invariant Subspaces, Linear Algebra Appl., 275-276 (1998), p. 161-177.

[13] J. Ferrer; F. Puerta; X. Puerta, Stratification and Bundle Structure of the Set of Conditioned Invariant Subspaces in the General Case, Systems and Control Letters, 48 (2003), p. 77-86.

[14] I. Gohberg; P. Lancaster; L. Rodman, Invariant Subspaces of Matrices with Applications. Wiley, Nova York (1986). 
Proceedings of the International Conference on Circuits, Systems, Signals

[15] M.A. Shayman, On the Variety of Invariant Subspaces of a Finite-dimensional Linear Operator, trans, Amer. Math. Soc., vol. 274 (2) (1982), p. 721-747.

[16] A. Tannenbaum, Invariance and System Theory: Algebraic and Geometric Aspects, LNM, n. 845, Springer (1981).

[17] W.M. Wonham, Linear Multivariable Control: A Geometric Approach, Springer, New York (1979). 